\title{
ACEPTABILIDAD DE PRE-ESCOLARES Y ESCOLARES A LA PROTEÍNA PURIFICADA DE PESCADO EN POLVO
}

\author{
Vanessa Bullón-Vela ${ }^{1, a}$, Gladys Valdiviezo ${ }^{1, b}$, Nelly Baiocchi ${ }^{1,2, c}$, Miguel Campos ${ }^{1, d}$, \\ Alejandro Llanos-Cuentas ${ }^{1,2, e}$, Theresa J. Ochoa ${ }^{1,2, f}$
}

\section{RESUMEN}

\begin{abstract}
Objetivos. Determinar la aceptabilidad de la proteína purificada de pescado (FPi) en bebidas a base de cereales y pseudocereales. Materiales y Métodos. El estudio se realizó en el Centro de Desarrollo Integral Familiar «Año Nuevo» del Programa Integral Nacional para el Bienestar Familiar (INABIF), del distrito de Comas en Lima. La FPi (0-4 g por ración) se administró diariamente durante tres meses mezclada en las bebidas a base de cereales y pseudocereales. Se midió la aceptabilidad de la FPi mediante pesado directo en niños menores de 6 años y por escala hedónica facial en escolares (6 a 16 años). Resultados. Se enroló a 158 participantes y se administró de manera supervisada 4211 desayunos. El aporte calórico de las bebidas a base de cereales y pseudocereales luego de adicionar la FPi estuvo entre 117 y $140 \mathrm{Kcal}$ y el aporte proteico entre 3,4 y 5,4 g por ración. La aceptabilidad en los niños mayores de 6 años de las bebidas suplementadas con la FPi (juntando los dos mayores puntajes) estuvo entre $55-74 \%$ para la avena, alrededor del $47 \%$ para la quinua y entre $40-58 \%$ para la kiwicha. Mientras que el $90 \%$ de los niños menores de 6 años consumieron más del $70 \%$ de lo ofrecido. Conclusiones. Se encontró una buena aceptabilidad de la FPi en las bebidas a base de cereal y pseudocereales en menores de 6 años. La FPi es una alternativa para mejorar el aporte de proteínas de origen animal en los niños, sobre todo en los preescolares.
\end{abstract}

Palabras claves: Alimentación; Hidrolisados de proteína; Desnutrición; Niños (fuente: DeCS BIREME).

\section{ACCEPTABILITY OF PURIFIED FISH-PROTEIN POWDER IN PRE-SCHOOL AND SCHOOL AGE CHILDREN}

\begin{abstract}
Objective. To determine the acceptability of purified fish protein (FPi) in cereal and pseudocereal-based drinks. Materials and Methods. The study was carried out at Centro de Desarrollo Integral Familiar "Año Nuevo" belonging to the INABIF Program in the district of Comas in Lima. FPi (0-4 g per serving) was administered daily for 3 months mixed with cereal and pseudocerealbased drinks. The acceptability of FPi was measured by direct weighing in children under 6 years of age and by the hedonic facial scale in school-age children. Results. One hundred and fifty-eight (158) participants were enrolled and 4,211 breakfasts were served. The caloric intake of the cereal and pseudocereal drinks, after adding the FPi, ranged between 117 and $140 \mathrm{Kcal}$, and protein intake ranged between $3.4 \mathrm{~g}$ and $5.4 \mathrm{~g}$ per serving. The acceptability of drinks supplemented with FPi in children older than 6 years of age (the 2 highest scores together) ranged from $55 \%$ to $74 \%$ for oats, around $47 \%$ for quinoa and $40 \%$ to $58 \%$ for "kiwicha" (amaranth). Whereas 90\% of children under 6 consumed more than $70 \%$ of the drinks offered. Conclusions. We found a good acceptability of $\mathrm{FPi}$ in cereal and pseudocereal beverages in children under 6 years of age. $\mathrm{FPi}$ is an alternative to improve the consumption of animal protein in the nutrition of children, especially in pre-schoolers.
\end{abstract}

Keywords: Alimentation; Protein hydrolysates; Malnutrition; Child (source: MeSH NLM).

\section{INTRODUCCIÓN}

La desnutrición es un problema de salud pública en Perú. En 2014, según la Encuesta Demográfica y de Salud (ENDES) el 14,6\% de los niños menores de cinco años padecían desnutrición crónica ${ }^{(1)}$. La desnutrición está relacionada a un incremento en el riesgo de enfermedades, rezago en el desarrollo cognitivo, motor y a un rendimiento escolar insatisfactorio ${ }^{(2,3)}$. A nivel mundial se considera que al menos 200 millones de niños menores de cinco años no llegan a alcanzar su potencial de desarrollo (2). En este contexto, un adecuado estado nutricional es fundamental durante la infancia ${ }^{(4)}$.

Las proteínas, constituidas por aminoácidos, desempeñan un rol importante para diversas funciones biológicas. Las

\footnotetext{
Universidad Peruana Cayetano Heredia. Lima, Perú;

Hospital Cayetano Heredia. Lima, Perú;

Nutricionista, magister en Alimentación, Nutrición y Metabolismo; ${ }^{\mathrm{b}}$ licenciada en Enfermería; ${ }^{\mathrm{c}}$ médico, magister en Nutrición Humana; ${ }^{\mathrm{d}}$ médico, Ph.D en Epidemiología; ${ }^{\mathrm{e}}$ médico infectólogo, Ph.D en Epidemiología; ${ }^{\mathrm{f}}$ médico infectólogo pediatra.

Recibido: 04/12/2017 Aprobado: 23/05/2018 En línea: 21/06/2018
}

Citar como: Bullón-Vela V, Valdiviezo G, Baiocchi N, Campos M, Llanos-Cuentas A, Ochoa TJ. Aceptabilidad de pre-escolares y escolares a la proteína purificada de pescado en polvo. Rev Peru Med Exp Salud Publica. 2018;35(2):234-40. doi: 10.17843/rpmesp.2018.352.3274. 
proteínas se degradan continuamente, produciéndose de manera conjunta el proceso de síntesis y degradación denominado recambio proteico. Este proceso obliga a su reposición dietética ${ }^{(5)}$; por lo tanto, una adecuada ingesta de proteínas en la dieta es necesaria para asegurar el crecimiento y desarrollo del niño ${ }^{(4,6)}$.

Los alimentos de origen animal contienen una alta proporción de proteínas, con una digestibilidad entre $95 \%$ y $98 \%{ }^{(7)}$, siendo el pescado una excelente fuente de aminoácidos y proteínas de fácil digestión ${ }^{(8,9)}$. La Comisión del Codex Alimentarius, creada por la Organización de la Naciones Unidas para la Agricultura y la Alimentación (FAO) y la Organización Mundial de la Salud (OMS), permite el uso de proteína de pescado en la alimentación infantil (10).

Los hidrolizados de proteínas pueden provenir de diversas fuentes y son utilizados en formulaciones infantiles ${ }^{(11)}$. Por otro lado, la inclusión de péptidos y nucleótidos en la dieta demuestran tener beneficios nutricionales y económicos ${ }^{(12,13)}$. Diversos estudios indican que el uso de los hidrolizados a base de pescado en la alimentación infantil es seguro y puede ser una alternativa para mejorar el estado nutricional de los niños debido a sus ingredientes bioactivos, elevada digestibilidad, fácil absorción y aminoácidos esenciales disponibles ${ }^{(9,13,14)}$. La proteína purificada del pescado (FPi) de Bluewave Marine Ingredients, contiene proteínas en forma de péptidos y nucleótidos, incluyendo ocho aminoácidos esenciales, como, lisina, metionina y cisteína ${ }^{(15)}$.

Si bien a nivel nacional el consumo de proteínas totales en la dieta logra superar el $100 \%$ de las recomendaciones nutricionales en niños menores de cinco años; el consumo de proteínas de origen animal no llega al $40 \%$ de las recomendaciones diarias en la sierra rural y trapecio andino ${ }^{(16)}$; por lo tanto, es necesario evaluar otras fuentes de proteínas de origen animal para poder utilizarse como un suplemento nutricional en estas regiones.

El presente estudio tiene como objetivo determinar la aceptabilidad de la FPi en el desayuno escolar de niños entre 3 y 16 años.

\section{MATERIALES Y MÉTODOS}

\section{DISEÑO Y POBLACIÓN DEL ESTUDIO}

Estudio comunitario de aceptabilidad realizado en el Centro de Desarrollo Integral Familiar (CEDIF) «Año Nuevo» perteneciente al Programa Integral Nacional para el Bienestar Familiar (INABIF) del distrito de Comas en Lima. La población de estudio fueron los niños del CEDIF. Los criterios de inclusión fueron ser mayor de tres años y recibir el desayuno proporcionado por el centro. No se enrolaron niños cuyos padres, o ellos, no aceptaran participar.

\section{MENSAJES CLAVE}

Motivación para realizar el estudio. La desnutrición continúa siendo un problema de salud pública en nuestro país, especialmente en zonas rurales; donde el aporte de proteínas de origen animal en la dieta no es adecuado.

Principales hallazgos. Se encontró una buena aceptabilidad de una proteína purificada de pescado (FPi) (proteína de origen animal) suplementado en bebidas en los niños menores de 6 años.

Implicancias. Es importante proporcionar un adecuado aporte de proteínas de alto valor biológico en la dieta y de bajo costo, siendo la FPi una alternativa de suplementación en bebidas a base de cereal y pseudocereales.

El estudio contó con la aprobación del comité institucional de ética de la Universidad Peruana Cayetano Heredia y el INABIF. Se realizaron diversas reuniones de presentación del estudio dirigidos a los padres de familias y miembros del CEDIF. La autorización de los padres o apoderados fue recogida mediante un consentimiento informado y un asentimiento por parte de los niños mayores de siete años.

\section{PROTEÍNA PURIFICADA DE PESCADO (FPi)}

La FPi (Bluewave Marine Ingredients, Proteicos Concentrados S.A.C. Ica, Perú) es un aislado de proteínas y péptidos de pescado de materia prima fresca, purificado mediante membranas. El producto es un polvo marrón claro con un ligero olor a proteínas y péptidos, que contiene $84 \%$ de proteína en $100 \mathrm{~g}$ de producto ${ }^{(15)}$.

\section{EVALUACIÓN DE LAACEPTABILIDAD}

Los niños recibieron el desayuno estándar proporcionado por el centro alimentario. Las bebidas a base de cereales (avena) y pseudocereales (quinua y kiwicha) con fruta (manzana o membrillo) fueron suplementadas con FPi (0, 2, 3 y $4 \mathrm{~g}$ por ración). Adicionalmente se acompañó de un sólido, como: camote sancochado o pan (con mantequilla o palta o queso o aceituna). En base a esta programación, se optó por suplementar la FPi en las bebidas a base de cereales, dado que los lácteos ya cuentan con un buen aporte proteico.

Se administraron de manera intercalada las preparaciones con y sin FPi durante un período de tres meses. Algunos días recibieron el desayuno suplementado con la FPi (diferentes concentraciones) y otros días sin esta. Para determinar la aceptabilidad de la FPi en los niños entre tres y seis años de edad, se analizó el consumo directo (diferencia del peso de la ración ofrecida y el peso sobrante). Se ofreció raciones de $250 \mathrm{~g}$; estas fueron pesadas por el mismo personal todos los días, en una balanza calibrada, bajo procedimientos estandarizados para el estudio y de acuerdo a las normas técnicas internacionales tales como 
ISO y las Normas Españolas de métodos de investigación de la sensibilidad gustativa ${ }^{(17)}$.

Se realizó el pesado de la taza con la bebida, antes y después de ofrecerla a los niños, para determinar el consumo. Luego de medir el volumen consumido, se determinó el porcentaje de niños que consumieron más del $70 \%$ de lo ofrecido (esta norma, ha fijado como límite mínimo aceptable al $70 \%$ de consumo, con el objetivo de asegurar la ingesta mínima requerida para satisfacer las necesidades del grupo de edad). Asimismo, se considera muy buena aceptabilidad cuando $90 \%$ o más de los niños consume $70 \%$ o más de lo ofrecido; buena aceptabilidad cuando $75 \%$ a $89 \%$ de los niños consumen $70 \%$ de lo ofrecido; regular, cuando el $60 \%$ y $74 \%$ consume $70 \%$ de lo ofrecido y mala aceptabilidad cuando menos del $60 \%$ de niños consume $70 \%$ de lo ofrecido ${ }^{(17)}$. Para los niños mayores de seis años se usó una escala hedónica facial que a través de cinco caritas, lo que permitía expresar el grado de preferencia del niño (puntaje uno, delicioso o le gusta muchísimo; puntaje dos, rico o bastante; puntaje tres, regular o más o menos; puntaje cuatro, feo o poco; puntaje cinco, horrible o nada) como método de evaluación sensorial, según la norma de la American Society for Testing and Material (ASTM International) (SIMS) ${ }^{(18)}$.

\section{DESCRIPCIÓN DEL ESTADO NUTRICIONAL Y SALUD}

Se realizó una evaluación médica, medición de hemoglobina y evaluación antropométrica al inicio del estudio para describir la condición de salud y nutrición basal de la población estudiada. Se realizó una evaluación médica, a cargo de un médico pediatra, para evaluar el estado de salud general de los participantes. Se tomó una muestra de sangre (punción digital) para determinar el nivel de hemoglobina mediante el HemoCue; para la determinación de anemia se tuvo en cuenta los puntos de corte especificados por la OMS 1986. Las mediciones antropométricas de peso y talla se realizaron de acuerdo a protocolo estandarizado al inicio del estudio ${ }^{(17)}$. Con la finalidad de evaluar el estado nutricional de la población se empleó el estándar internacional de la OMS 2006 para niños menores de cinco años para determinar el puntaje $Z$ talla/ edad y peso/talla; mientras que para los niños mayores de cinco años se utilizó el puntaje $Z$ talla/edad y el puntaje $Z$ del índice de masa corporal (IMC), según OMS 2007.

\section{ANÁLISIS ESTADÍSTICO}

El tamaño muestral fue por conveniencia, se enroló al total de escolares que asistían en el turno de la mañana y que aceptaron participar. Las variables dependientes se construyeron de manera dicótoma debido a su distribución asimétrica. En la escala hedónica se utilizaron las categorías uno y mayor de 1. Mientras tanto, para la cantidad sobrante se usaron las categorías sobrantes menor de cinco $\mathrm{g} o$ sobrantes de cinco o más gramos. En el análisis del efecto del tipo de menú sobre las variables dependientes, se aplicaron modelos lineales generalizados teniendo como factores candidato iniciales al tipo de acompañante (FACOM en tres categorías: manzana, membrillo y otros), la concentración de FPi (PROTEINA, continua), el día del mes (FECHADM, entera de 1 a 31), el día de la semana (FECHADW, categórica de 1 a 7) y dos términos de interacción (FACOM ${ }^{\star}$ PROTEINA, y FECHADM*FECHADW). Estos términos se sometieron a una selección progresiva (Stepwise) con regresión logística múltiple. Los términos que resultaron seleccionados se verificaron con un modelo lineal mixto, agregando términos de aula y de alumno dentro del aula. Los datos se analizaron con el paquete estadístico $R$ (con los paquetes EPICALC y LME4). Se calculó la edad en días y los índices antropométricos como puntaje $Z$ usando los coeficientes LMS de la norma OMS 2006, considerando como normal de $-2 \mathrm{a}+2 \mathrm{DE}$.

\section{RESULTADOS}

Se enrolaron 158 escolares. La edad promedio fue de ocho \pm dos años (58,2 \% menores de siete años) y 50,6 \% fueron de sexo masculino. El principal diagnóstico médico basal fue la presencia de caries dentales $(27,8 \%)$ y anemia (30,8\%); siendo el grupo con mayor frecuencia el $\leq$ seis años (Tabla 1). En relación al diagnóstico del estado nutricional, se encontró un $12 \%$ de déficit de talla para la edad (desnutrición crónica, <-2 DE) con mayor compromiso en el grupo de siete a nueve años. No se encontró déficit de IMC para la edad, pero si un exceso de peso (>+2 DE) en un $15,2 \%$, principalmente en los niños entre siete y nueve años (Tabla 2).

Se brindó de manera supervisada 4211 desayunos (2019 a niños tres a seis años y 2192 a niños seis a 16 años); el promedio de raciones por niño fue de 27 . El aporte calórico de los cereales sin adicionar la FPi osciló entre 110 a 130 Kcal con un aporte de proteína de 1,7 a 2,1 g. Al adicionar la FPi, el aporte calórico estuvo entre 117,3 y 143,2 Kcal y el aporte de proteína entre 3,4 y 5,4 g.

Tabla 1. Características demográficas y clínicas basales de los niños y adolescentes enrolados

\begin{tabular}{ll}
\hline Característica (n = 158) & $\mathbf{n ~ ( \% ) ~}$ \\
\hline Edad (años) & $92(58,2)$ \\
3 a 6 & $28(17,7)$ \\
7 a 9 & $38(24,1)$ \\
10 a 16 & $80(50,6)$ \\
Sexo, masculino & \\
Diagnóstico médico basal & $44(27,8)$ \\
$\quad$ Caries dental & $11(7,0)$ \\
Otros & $7(4,4)$ \\
Infección respiratoria alta & $2(1,3)$ \\
\hline Infección de piel o tejidos blandos & $2(1,3)$ \\
\hline Otras lesiones de piel & $1(0,6)$ \\
Asma & $1(0,6)$ \\
Infección respiratoria baja & \\
Frecuencia de anemia (años) & $20(27,4)$ \\
3 a 6 & $10(38,5)$ \\
7 a 9 & $11(32,4)$ \\
\hline 10 a 16 &
\end{tabular}


Tabla 2. Diagnóstico nutricional basal de los niños y adolescentes enrolados

\begin{tabular}{|c|c|c|c|c|}
\hline \multirow{2}{*}{ Grupo etario (años) } & \multicolumn{2}{|c|}{ T/E n (\%) } & \multicolumn{2}{|c|}{ IMC n (\%) } \\
\hline & Normal & Talla baja & Normal & Exceso de peso \\
\hline Tres a seis & $84(91,3)$ & $8(8,7)$ & $83(90,2)$ & $9(9,8)$ \\
\hline Siete a nueve & $23(82,1)$ & $11(17,9)$ & $19(67,9)$ & $9(32,1)$ \\
\hline Diez a 16 & $32(84,2)$ & $6(15,8)$ & $32(84,2)$ & $6(15,8)$ \\
\hline Total & $139(88,0)$ & $19(12,0)$ & $134(84,8)$ & $24(15,2)$ \\
\hline
\end{tabular}

T/E: talla para edad, IMC: indice de masa corporal para la edad.

La aceptación de la FPi en el desayuno escolar, evaluada mediante la escala hedónica facial, fue adecuada. En el caso de la avena entre el $44 \%$ y $55 \%$ de los niños indicaron que estaba delicioso o que les gustaba muchísimo (puntaje uno) tanto en su preparación con manzana o membrillo (Tabla 3). Del mismo modo, en las bebidas a base de kiwicha y quinua, entre un $30 \%$ a $51 \%$ de los niños indicaron que estaba delicioso o que les gustaba muchísimo. Al comparar este resultado con la aceptabilidad del cereal y pseudocereal sin agregar la FPi $(0 \mathrm{~g})$, esta osciló entre un $42 \%$ y $61 \%$ aproximadamente. En relación al peor puntaje (puntaje cinco) de los cereales evaluados; la mayor desaprobación para la avena con manzana o membrillo suplementada con FPi se halló a mayores concentraciones de $\mathrm{FPi}$, para tres g $(17,1 \%$ a $23,5 \%)$ y cuatro g $(21,5 \%$ a $24,1 \%)$ respectivamente; en las bebidas a base de quinua con manzana o membrillo suplementada con tres g de FPi fue de $31,3 \%$ y $14,3 \%$ respectivamente; mientras que, para la kiwicha con membrillo fue de $32,8 \%$ a dos g, 31,6 \% a tres g y del $29 \%$ para cuatro g (Tabla 3). El análisis multivariado mostró para la escala hedónica facial un efecto positivo de la FPi (es decir mayor puntaje o menor aceptabilidad) después de ajustar por el día de la semana y el día del mes ( $p<0,001)$; observando que a mayor concentración de FPi, menor aceptabilidad.

En el caso de la aceptación de la FPi evaluada mediante consumo (diferencia entre peso antes y después de la administración del alimento), esta fue muy buena.

Tabla 3. Aceptabilidad de las bebidas a base de cereales y pseudocereales a diferentes concentraciones de la proteína purificada de pescado, según escala hedónica facial en escolares mayores de seis años

\begin{tabular}{|c|c|c|c|c|c|c|c|c|}
\hline \multicolumn{2}{|c|}{ Tipo de bebida } & \multicolumn{2}{|c|}{ FPi } & \multicolumn{5}{|c|}{ Puntaje de escala hedónica facial n (\%) } \\
\hline Sustrato & Acompañante & $\begin{array}{c}\text { Gramos } \\
\text { por ración }\end{array}$ & Frecuencia & 1 & 2 & 3 & 4 & 5 \\
\hline \multirow{8}{*}{ Avena } & \multirow{4}{*}{ Manzana } & 0 & 122 & $74(60,7)$ & $20(16,4)$ & $19(15,6)$ & $2(1,6)$ & $7(5,7)$ \\
\hline & & 2 & 113 & $56(49,6)$ & $15(13,3)$ & $12(10,6)$ & $13(11,5)$ & $17(15,0)$ \\
\hline & & 3 & 129 & $65(50,4)$ & $18(14,0)$ & $14(10,8)$ & $10(7,8)$ & $22(17,1)$ \\
\hline & & 4 & 93 & $41(44,1)$ & $10(10,8)$ & $15(16,1)$ & $7(7,5)$ & $20(21,5)$ \\
\hline & \multirow{4}{*}{ Membrillo } & 0 & 23 & $11(47,8)$ & $5(21,7)$ & $4(17,4)$ & $1(4,3)$ & $2(8,7)$ \\
\hline & & 2 & 97 & $53(54,6)$ & $19(19,6)$ & $7(7,2)$ & $9(9,3)$ & $9(9,3)$ \\
\hline & & 3 & 119 & $55(46,2)$ & $15(12,6)$ & $15(12,6)$ & $6(5,0)$ & $28(23,5)$ \\
\hline & & 4 & 29 & $16(55,2)$ & $1(3,4)$ & $5(17,2)$ & $0(0,0)$ & $7(24,1)$ \\
\hline \multirow{6}{*}{ Quinua } & \multirow{2}{*}{ Manzana } & 0 & 36 & $21(58,3)$ & $6(16,7)$ & $6(16,7)$ & $2(5,6)$ & $1(2,8)$ \\
\hline & & 3 & 32 & $11(34,4)$ & $4(12,5)$ & $6(18,8)$ & $1(3,1)$ & $10(31,3)$ \\
\hline & \multirow{4}{*}{ Membrillo } & 0 & 28 & $16(57,1)$ & $4(14,3)$ & $5(17,9)$ & $1(3,6)$ & $2(7,1)$ \\
\hline & & 2 & 58 & $25(43,1)$ & $4(6,9)$ & $7(12,1)$ & $4(6,9)$ & $18(31,0)$ \\
\hline & & 3 & 21 & $7(33,3)$ & $3(14,3)$ & $3(14,3)$ & $5(23,8)$ & $3(14,3)$ \\
\hline & & 4 & 26 & $13(50)$ & $3(11,5)$ & $4(15,4)$ & $3(11,5)$ & $3(11,5)$ \\
\hline \multirow{4}{*}{ Kiwicha } & \multirow{4}{*}{ Membrillo } & 0 & 111 & $47(42,3)$ & $14(12,6)$ & $19(17,1)$ & $12(10,8)$ & $19(17,1)$ \\
\hline & & 2 & 63 & $19(30,2)$ & $6(9,5)$ & $9(14,3)$ & $8(12,7)$ & $21(33,3)$ \\
\hline & & 3 & 57 & $29(50,9)$ & $4(7,0)$ & $4(7,0)$ & $2(3,5)$ & $18(31,6)$ \\
\hline & & 4 & 60 & $26(43,3)$ & $6(10,0)$ & $6(10,0)$ & $4(6,7)$ & $18(30,0)$ \\
\hline Leche & Otro & 0 & 975 & $577(59,1)$ & $183(18,7)$ & $134(13,7)$ & $27(2,8)$ & $54(5,5)$ \\
\hline
\end{tabular}

Escala hedónica facial expresada en puntajes de 1 a 5: puntaje 1: delicioso o le gusta muchísimo; Puntaje 2: rico o bastante; Puntaje 3: regular o más o menos; Puntaje 4: feo o poco; Puntaje 5: horrible o nada.

FPi: Proteína purificada de pescado

* Las bebidas fueron suplementadas con $\mathrm{FPi}(0,2,3$ y $4 \mathrm{~g}$ por ración) 
Podemos observar que más del $90 \%$ de niños consumen más del $90 \%$ de la ración (es decir menos de $25 \mathrm{~g}$ de sobrante); por lo tanto, la mayoría de los niños tuvieron un consumo por encima del $70 \%$. Los niños menores de seis años consumieron entre de $220 \mathrm{~g}$ y $240 \mathrm{~g}$ de cereal aproximadamente ( $\pm 10 \%$ ) y en todos los casos, a diferentes concentraciones de la FPi, el consumo fue bastante homogéneo (Tabla 4). En general, el sobrante fue de aproximadamente cinco g para la avena y quinua, mientras que alrededor de diez $\mathrm{g}$ en el caso de la kiwicha (Figura 1). Sin embargo, estas cantidades representan menos del $5 \%$ de la cantidad inicial de la ración. El análisis no encontró un efecto de la FPi en la cantidad sobrante, ajustando por el cereal y pseudocereal, el día de la semana y el día del mes $(p=0,12)$.

Hay que tomar en cuenta que el diseño tiene como excluyentes a la leche y la proteína. Asimismo, no se trata de un diseño factorial puro (los tratamientos no han sido asignados por un proceso aleatorio formal). En particular, los intervalos de $95 \%$ de confianza indican que sobre un porcentaje de satisfacción hipotético de $50 \%$, el efecto posible de cada gramo de concentración FPi se encuentra entre $+4 \%$ y $+14 \%$ niños satisfechos (con puntaje hedónico de dos o menos), y entre $-19 \%$ y $+2 \%$ niños satisfechos (con sobrante menor de cinco g).

\section{DISCUSIÓN}

Este es el primer estudio en la que se mide la aceptabilidad de la FPi suplementada en desayunos escolares. Se emplearon dos metodologías para evaluar de aceptabilidad según la edad de los niños. Las normas técnicas internacionales tales como ISO y las normas españolas de métodos de investigación de la sensibilidad gustativa ${ }^{(17)}$ establecen varios parámetros para medir aceptabilidad. Uno de ellos es la medición de consumo, usada principalmente en niños pequeños. Luego de medir el volumen consumido, se determina el porcentaje de niños que consumen más del $70 \%$ de lo ofrecido. Según esta norma, se considera muy buena aceptabilidad cuando $90 \%$ o más de los niños consume $70 \%$ o más de lo ofrecido. Por lo tanto, la FPi mezclada con los cereales y pseudocereales evaluados tiene una muy buena aceptabilidad. Otra metodología incluye la evaluación sensorial. Es decir, la determinación de las características organolépticas del producto, tales como: opinión sobre apariencia, consistencia, olor, sabor, sabores extraños y aceptación en general.

Normalmente la evaluación de las características organolépticas se realiza con un panel de adultos; en este estudio, nosotros evaluamos la aceptabilidad en niños

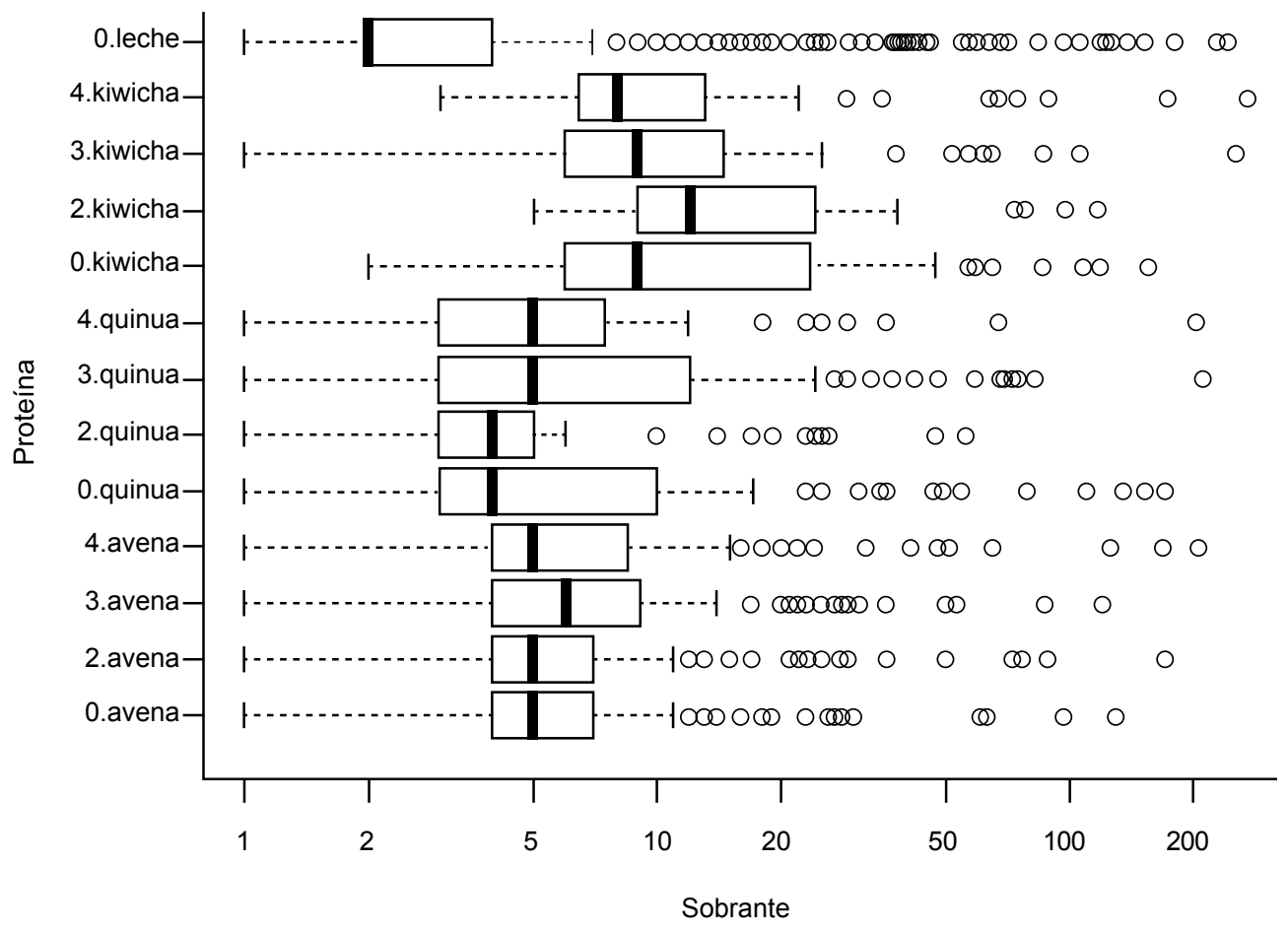

Figura 1. Distribución de la cantidad sobrante (gramos) de acuerdo con la concentración de proteína purificada de pescado en los diferentes cereales y pseudocereales

En el eje $\mathrm{Y}$ está la cantidad de proteína purificada de pescado ( $\mathrm{FPi})$ : 0.avena, es $0 \mathrm{~g}$ de $\mathrm{FPi}$ en la preparación con avena; 2.avena es $2 \mathrm{~g}$ de FPi en la preparación de avena, 3.quinua, es $3 \mathrm{~g}$ de FPi en la preparación de quinua, y así sucesivamente. En el eje $\mathrm{X}$ está el sobrante en gramos de cada ración. 
Tabla 4. Consumo de bebidas suplementadas con o sin proteína purificada de pescado en niños menores de 6 años

\begin{tabular}{|c|c|c|c|c|}
\hline \multicolumn{2}{|c|}{ Tipo de bebida } & FPi g* & $n$ & Consumo $^{\dagger}$ \\
\hline \multirow{8}{*}{ Avena } & \multirow{4}{*}{ Manzana } & 0 & 112 & $230(25)$ \\
\hline & & 2 & 104 & $224(23)$ \\
\hline & & 3 & 119 & 227 (29) \\
\hline & & 4 & 86 & $226(23)$ \\
\hline & \multirow{4}{*}{ Membrillo } & 0 & 21 & 227 (22) \\
\hline & & 2 & 89 & $223(23)$ \\
\hline & & 3 & 109 & $221(23)$ \\
\hline & & 4 & 27 & $239(43)$ \\
\hline \multirow{6}{*}{ Quinua } & \multirow{2}{*}{ Manzana } & 0 & 33 & 227 (23) \\
\hline & & 3 & 29 & $245(46)$ \\
\hline & \multirow{4}{*}{ Membrillo } & 0 & 26 & $227(22)$ \\
\hline & & 2 & 53 & $233(30)$ \\
\hline & & 3 & 19 & $228(23)$ \\
\hline & & 4 & 24 & $228(24)$ \\
\hline \multirow{4}{*}{ Kiwicha } & \multirow{4}{*}{ Membrillo } & 0 & 102 & $218(22)$ \\
\hline & & 2 & 58 & $220(27)$ \\
\hline & & 3 & 52 & $236(30)$ \\
\hline & & 4 & 55 & 231 (29) \\
\hline Leche & otro & 0 & 899 & $226(24)$ \\
\hline
\end{tabular}

FPi: proteína purificada de pescado, $\mathrm{n}$ : número de raciones

* Las bebidas fueron suplementadas con $\mathrm{FPi}(0,2,3$ y 4 g por ración)

† El consumo en gramos se expresa en media y desviación estándar.

utilizando un método más sencillo. Según este método, la aceptación (puntaje uno) fue en general mayor del $50 \%$. No obstante, cabe señalar que la aceptación de los cereales y pseudocereales, sin adicionar la $\mathrm{FPi}$, estuvo entre $40 \%$ y $60 \%$; de manera similar, la aceptación de la leche estuvo alrededor de un $60 \%$; indicando que con el desayuno estándar (sin suplementar con la FPi), la aceptabilidad fue similar. Sin embargo, el análisis multivariado mostró que la aceptabilidad fue menor a mayor concentración de la FPi suplementada. Esto podría deberse a las características propias del suplemento; los niños refirieron en algunos casos, sentir olor y sabor a pescado, lo que pudo influenciar su percepción.

Los resultados mostraron que se pudo incrementar el aporte de proteínas de alto valor biológico a través del uso de la FPi con una adecuada aceptabilidad. Además, no se reportó eventos adversos relacionados al consumo de esta proteína. Estos resultados son similares al estudio aleatorizado, paralelo, multicéntrico, doble ciego realizado por Nesse et al. que evaluaron el consumo diario de un hidrolizado de proteína de pescado en 438 niños malnutridos entre seis a ocho años de edad. Los niños fueron aleatorizados en tres grupos. Al grupo control, se les brindó una bebida a base de chocolate; mientras que, a los otros grupos se suplementó la misma bebida, pero con tres $\mathrm{g}$ y seis $\mathrm{g}$ de proteína por día. Se analizaron datos antropométricos, muestras de sangre y de orina. No se registraron eventos adversos ni efectos negativos en el peso y talla, demostrando que su uso es seguro en la alimentación de niños malnutridos (14). Brinkman et al. evaluaron la aceptabilidad y tolerancia de concentrados de proteínas de pescado en la nutrición de niños preescolares, no encontrando efecto en la ganancia de peso ${ }^{(19)}$.

Nuestro grupo de investigación previamente evaluó la seguridad, aceptabilidad y eficacia de la FPi en la nutrición de niños menores de tres años. Un total de 441 niños entre seis y 36 meses fueron aleatorizados a recibir $\mathrm{FPi}$ reemplazando el $50 \%$ de la proteína en el almuerzo versus la dieta con la proteína estándar durante seis meses. El crecimiento de los niños fue similar, así como el consumo; no reportándose eventos adversos. El costo de los almuerzos con FPi fue entre $20 \%$ a $40 \%$ menor que la dieta estándar con otras proteínas de origen animal ${ }^{(20)}$. De manera similar, el presente estudio en niños mayores de tres años mostró una adecuada aceptabilidad del uso de proteína purificada de pescado en la alimentación de los niños, sobre todo en los menores de seis años.

Una de las limitaciones de este estudio es que se suplemento la FPi sólo en tres bebidas a base de cereales y pseudocereales. Sin embargo, los productos evaluados forman parte de los productos básicos en la alimentación en Perú, según la Encuesta Nacional de Presupuestos Familiares (ENAPREF) 2008 - 2009. Una segunda limitación fue que el preparado formaba parte del desayuno, por lo que los niños más pequeños consumieron todo, no necesariamente por el gusto, sino por la necesidad (hambre). Otra limitación es que la escala hedónica usada tiene los puntajes al revés de como normalmente se reporta en la literatura; usualmente el puntaje más alto es de mayor aceptabilidad. Sin embargo, creemos que la variación en el orden no invalida los resultados del estudio, dado que la ficha usada incluía caritas y un breve texto, más no el puntaje (ejemplo: imagen de una carita feliz y debajo el texto "delicioso o me gusta muchísimo»). El puntaje fue agregado por los investigadores al ingresar la información a la base de datos.

Pese a estas limitaciones nuestro estudio tiene algunas fortalezas, incluyendo la rigurosidad de las mediciones para determinar el consumo (se pesaron más de 2000 raciones antes y después de adminístrales a los niños). Adicionalmente, se incluyeron en la evaluación un número relativamente grande de niños, los cuales recibieron en múltiples oportunidades las raciones suplementadas o no con la FPi. La mayoría de los estudios de aceptabilidad se realizan con menor número de sujetos y en pocos días de observación.

\section{CONCLUSIONES}

El presente estudio demostró una buena aceptabilidad de la FPi suplementado en el desayuno escolar, sobre todo en los menores de seis años. Se recomienda el uso de la FPi en la alimentación de niños para mejorar el aporte 
proteico sobre todo en zonas rurales de la selva y sierra del Perú, donde la accesibilidad a proteínas de origen animal es menor. Se sugiere realizar estudios complementarios considerando los factores culturales y sociales en estas poblaciones.

Agradecimientos: Al INABIF, CEDIF Año nuevo, a los padres de familia y a los voluntarios participantes en el presente estudio.
Fuentes de financiamiento: Fondo de las Américas (FONDAM) y Proteicos Concentrados S.A.C. Perú.

Conflicto de intereses: Los autores que han participado en este estudio declaran que no tienen acceso financiero o conflicto de intereses. La empresa Proteicos Concentrados S.A.C. que financió parcialmente el estudio es la que fabrica y comercializa la proteína purificada de pescado (FPi); sin embargo, no participaron en el diseño, ejecución, análisis ni redacción del manuscrito del estudio.

\section{REFERENCIAS BIBLIOGRÁFICAS}

1. Encuesta Demográfica y de Salud Familiar 2014. Encuesta Demográfica y Salud Familiar. Instituto Nacional de Estadística e Informática. 2015; 40.

2. Grantham-McGregor $S$, Cheung $Y B$, Cueto S, Glewwe P, Richter L, Strupp B, et al. Developmental potential in the first 5 years for children in developing countries. Lancet. 2007;369(9555):60-70.

3. Aguilar C, Knapp M, Moscoso MS, Pollitt E. Pontificia Universidad Católica del Perú. 2010;28(2).

4. Scott MI. Feeding and Nutrition of Infants and Young Children: Guidelines for the WHO European Region, With Emphasis on the Former Soviet Countries [Book Review]. J Hum Lact. 2001;17(3):265-6.

5. Gil Hernandez A. Tratado de nutrición. 2da edición. Madrid: Médica Panamericana; 2010.

6. World Health Organization. Infant and young child feeding Model Chapter for textbooks for medical students and allied health professionals. Geneva: World Health Organization; 2011.

7. Moughan PJ. Protein: Digestion, Absorption and Metabolism. Encycl Food Heal. 2016. 524-9.

8. Kristinsson HG, Rasco BA. Fish Protein Hydrolysates: Production, Biochemical, and Functional Properties. Crit Rev Food Sci Nutr. 2000;40(1):43-81.

9. Chalamaiah M, Dinesh Kumar B, Hemalatha R, Jyothirmayi T. Fish protein hy- drolysates: Proximate composition, amino acid composition, antioxidant activities and applications: A review. Food Chem. 2012;135(4):3020-38. doi: 10.1016/j. foodchem.2012.06.100.

10. Organización Panamericana de la Salud. Seguridad Alimentaria y Nutricional en la comunidad. Washington, DC: Organización Panamericana de la Salud; 2002.

11. Moreno Villares JM, Oliveros Leal L, Galiano Segovia MJ. Cómo enriquecer la alimentación del lactante: Uso de los modulos nutricionales. Acta Pediatr Esp. 2003;61(8):406-12.

12. De Pee S, Bloem MW. Current and potential role of specially formulated foods and food supplements for preventing malnutrition among 6-23 month old children and treating moderate malnutrition among 6-59 months old children. Food Nutr Bull. 2009;30(3):1-40.

13. Nesse KO, Nagalakshmi AP, Marimuthu P, Singh M. Efficacy of a fish protein hydrolysate in malnourished children. Indian J Clin Biochem. 2011;26(4):360-5.

14. Nesse KO, Nagalakshmi AP, Marimuthu P, Singh M, Bhetariya PJ, Ho M, et al. Safety evaluation of fish protein hydrolysate supplementation in malnourished children. Regul Toxicol Pharmacol. 2014;69(1):1-6.

15. Bluewave Peru Food Protein Products for Human Consumption [Internet]. Ica: bluewaveperu.com; 2017 [acceso 5 marzo 2017] Disponible en: http://www. bluewaveperu.com/food.htm
16. Miranda M, Rojas C, Barboza J, Riega V, Valenzuela $\mathrm{R}$, Cavero $\mathrm{S}$, et al. Tendencias en el cosumo de energía y nutrientes de niños peruanos menores de 5 años en el período 1997-2001. Rev Peru Med Exp Salud Publica. 2004;21(4):240-52.

17. Asociación Española de Normalización y Certificación. UNE 87-003-95: Análisis sensorial. Metodología. Método de investigación de la sensibilidad gustativa. Tomo I Alimentación. Madrid: AENOR;1997.

18. SIMS Sensory Software. Sensory and Consumer Smiley Face Testing With Children. 2001;11:273-83.

19. Brinkman GL, Sharadambal B, Madhave $\mathrm{V}$. A feeding trial of fish protein concentrate with preschool children. Am J Clin Nutr. 1970;23(4):395-9. doi: 10.1093/ ajcn/23.4.395.

20. Ochoa TJ, Baiocchi N, Valdiviezo G, Bullon V, Campos M, Llanos-Cuentas A. Evaluation of the efficacy, safety and acceptability of a fish protein isolate in the nutrition of children under 36 months of age. Public Health Nutr. 2017, 14;20:2819-26.

Correspondencia: Theresa J. Ochoa, MD Dirección: Universidad Peruana Cayetano Heredia, Av. Honorio Delgado 430. San Martin de Porres, Lima 31, Perú

Teléfono: (511)3190000 Anexo: 2715

Correoelectrónico: Theresa.Ochoa@upch.pe ; Theresa.J.Ochoa@uth.tmc.edu 